\title{
POLICY BASED ACCESS ROUTER SELECTIONS AND CONTEXT TRANSFERS IN MOBILE IP NETWORK
}

\author{
Ram Gopal .L, Man Li \\ Nokia Research Center, 5 Wayside Road, Burlington, MA 01803, ram.gopal@nokia.com, \\ man.m.li@nokia.com
}

\begin{abstract}
In mobile Internet Protocol (IP) networks, a handover occurs when a mobile node $(\mathrm{MN})$ moves from one cell to another. The result of a handover is that the mobile node connects to the network through a new access router (AR). A handover may occur between access routers of the same or different administrative domains. In both cases, to provide seamless handover the new access router needs to be selected first, then the information related to the mobile node, also called context, has to be transferred from the old access router to the new access router. This operation is called context transfer. In this paper we propose a policy-based scheme for access router selection and seamless context transfer mechanism. We also provide an analysis to show that this is efficient, secure and does not require significant changes to the existing network infrastructure.
\end{abstract}

Key words: Internet Protocol, Mobile Node, Access Router, Policy, and Autonomous System

\section{INTRODUCTION}

Internet Protocol (IP) is being adopted as transport protocol in All IP wireless network. It is expected that new wireless devices will have IP capabilities. IP provides connection less datagram service, the complete intelligence lies at the endpoints of the system. In order to serve the mobile node, in wireless network the last hop router (Access router) maintains information pertaining to each mobile node and is called MN context. A handover may occur between access routers of the same or different

The original version of this chapter was revised: The copyright line was incorrect. This has been corrected. The Erratum to this chapter is available at DOI: 10.1007/978-0-387-35620-4_43 
administrative domains. In both cases, to provide seamless handover the new access router needs to be selected first, then the information related to the mobile node, also called context, has to be transferred from the old access router to the new access router. This operation is called context transfer.

In this paper we propose a policy-based access router selection and context transfer approach that is efficient, secure and that does not require significant additional functionalities being built into access routers. The policy-based approach contains two steps. The first step is the access router selection process where a policy server computes a list of possible ARs that may serve an $\mathrm{MN}$ in its domain. The policy server then informs the $\mathrm{MN}$ this list. The second step involves the actual context transfer. Details of the two steps are described in the following Sections. This paper is organized as follows. First we describe the overview of wireless network, key functional elements and the context transfer issues. In section 2 we propose a policy based access router selection process. Section 3 presents two policy based context transfer procedures. Finally sections 4 and 5 provide analysis and conclusion of the proposed solution respectively.

\subsection{Overview}

Figure 1 shows the basic building blocks of a wireless network with main functional entities and interfaces.

- The Radio Access Network (RAN) handles all radio related functionality.

- The Core Network $(\mathrm{CN})$ is responsible for switching and routing calls, as well as for handling the data connection to external networks.

- The user's Mobile Node (MN) interfaces to the network via the radio interface.

- The Base Station (BS) implements layer 2 functions and connect mobile nodes to the access router. Air interface related protocols are implemented at BS.

- The Radio Network Controller (RNC) provides paging server functions and serves as an anchor point for micro mobility and radio network gateway control functions

- The Access Router (AR) is the last mile router connecting the mobile node to the IP network. Access router maintains context information pertaining to each mobile node. The access router provides IP (Internet Protocol) connectivity to the MN and servers as a point of attachment to the $\mathrm{MN}$.

- The Servicing GPRS Service Node (SGSN) serves the operator's subscribers and controls the usage of the 3G networks. The SGSN 
control part consists of the Mobility Management functions and the Session Management control functions.

- The Gateway GPRS Service Node (GGSN) provides inter-working with external packet-switched networks such as the public Internet.

- The Mobile switching Center (MSC) and Visitor Location Register (VLR) are responsible for switching voice calls and for providing connectivity and service related to telephone system.

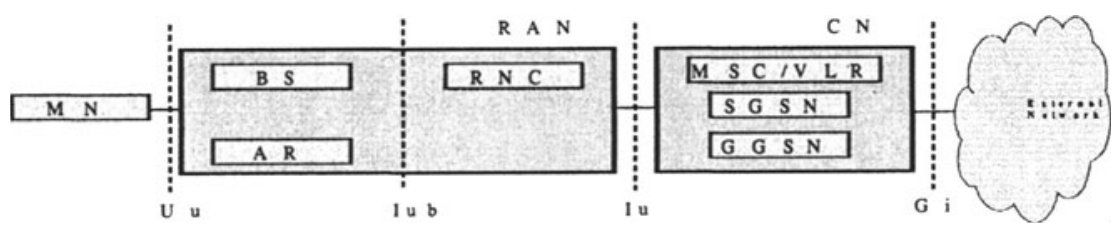

Figure 1 High-level block diagram of a wireless network

In all IP networks, the base station (BS) performs layer 2 functions. An Access router (AR) provides IP connectivity to the Mobile nodes and serves as a point of attachment through which the Mobile node communicates to the Internet. After the $\mathrm{MN}$ is turned $\mathrm{ON}$, the initial authentication and authorization process takes place. As a result of this process, security associations are created between $\mathrm{MN}$ and AR. Apart from this, the access router also maintains certain context information pertaining to each $\mathrm{MN}$. This includes header compression context, QoS context and Security context etc. When the $\mathrm{MN}$ moves from one cell to another cell, the point of attachment changes. The roaming decision is made to ensure that the mobile node is authorized to roam through the new access routers. After this, the complete context is relocated from the old access router to the new access router. This transfer must satisfy the requirements of time critical real time application. Current or proposed solutions [2][3] are based on moving the complete intelligence to the network elements, i.e., access routers. Each access router must discover candidate access routers for possible handover, select the target access router for actual handover based on the capabilities of the mobile node, authenticate the target access router and finally perform the context transfer. Specifically, each access router performs the following functions:

- Contacting the respective Home Agent server

- Contacting the Home Authentication Authorization and Accounting (AAA) server

- Interpreting the static subscription profile of the mobile node

- Authenticating and authorizing the neighboring access routers 
- Interpreting the static capability of the neighboring access routers (and/or)

- Moving the static capabilities of the mobile node to the access routers (and/or)

- Performing some pre-context activities before the actual context transfer

- Finally transferring the context to the new access router

The above-mentioned functions are in addition to the main responsibilities of an access router, i.e., to route IP packets based on subscriber information and/or to perform metering and monitoring for charging and management purposes. To incorporate the above functionality requires a radical change to the design of many access routers that are already in use in Internet and adds complexity to peering relationship between adjacent network service providers. The following are the potential shortcomings:

- Currently there is no common mechanism for two access routers to exchange mobile specific context information across two autonomous systems (AS).

- For security reasons, network operators do not want to expose their capabilities or capacity of their network elements. If one of the router is compromised the whole system is likely to get compromised. Yet current or proposed solutions require routers to expose their capabilities to other routers in same or different domains

- Moving the intelligence to the access router is a security issue. Control and update distributed information are potential problems. In strictly protected networks such as Telecom networks, this may be less important. But IP networks are not as easy to protect as Telecom networks.

- There are no automatic schemes where routers can authenticate each other. They may rely on public key based mechanisms. But these require more time to be widely deployed.

- The router selection rules or algorithms are installed on all access routers or Mobile Nodes. This may increase the cost of both access routers and mobile nodes and impact router performance. In addition, a simple change of the selection rules requires updating on all routers or mobile nodes. 


\section{POLICY BASED ACCESS ROUTER SELECTION}

Figure 2 shows an example of the context transfer framework and the target access router selection process. When policy based management approach is adopted, access routers are policy targets. On boot up, they report their capabilities (QoS, Security etc) to the policy server through the COPS protocol [3]. The policy server then downloads the corresponding policy to the AR according to the reported capabilities with various Policy Information Bases (PIB) [4][5]. Therefore the policy server has all the information about AR capabilities in the administrative domain. In addition the policy server can retrieve information relating to the base station from the database of network management systems. The information includes, for example, which access router a base station is connected to. In Figure 2, MN is currently in Autonomous System (say AS1) and is communicating with a server in Correspondent Node $(\mathrm{CN})$. The static capabilities of the $\mathrm{MN}$ are stored in the Authentication, Authorization and Accounting server (say AAA1). The policy server can retrieve this information from the AAA server of the MN home network. In the following, we describe the problem of inter domain handover which is more complicated than intra domain handovers. For example, when the MN is in the AS1, the Policy server in AS1 (say PS1) retrieves the static capabilities of the MN from AAA1 server. The dynamic capabilities (or negotiated profiles) of the $\mathrm{MN}$ are kept with the access router AR1 that is currently serving the MN. When the MN moves toward AS2, it receives identification information on a broadcast channel which may contain link layer information of Base Station (say BS2) or IP address of AR2 or AS number associating some link local address or any combination of the above information. MN forwards the information to AR1. 


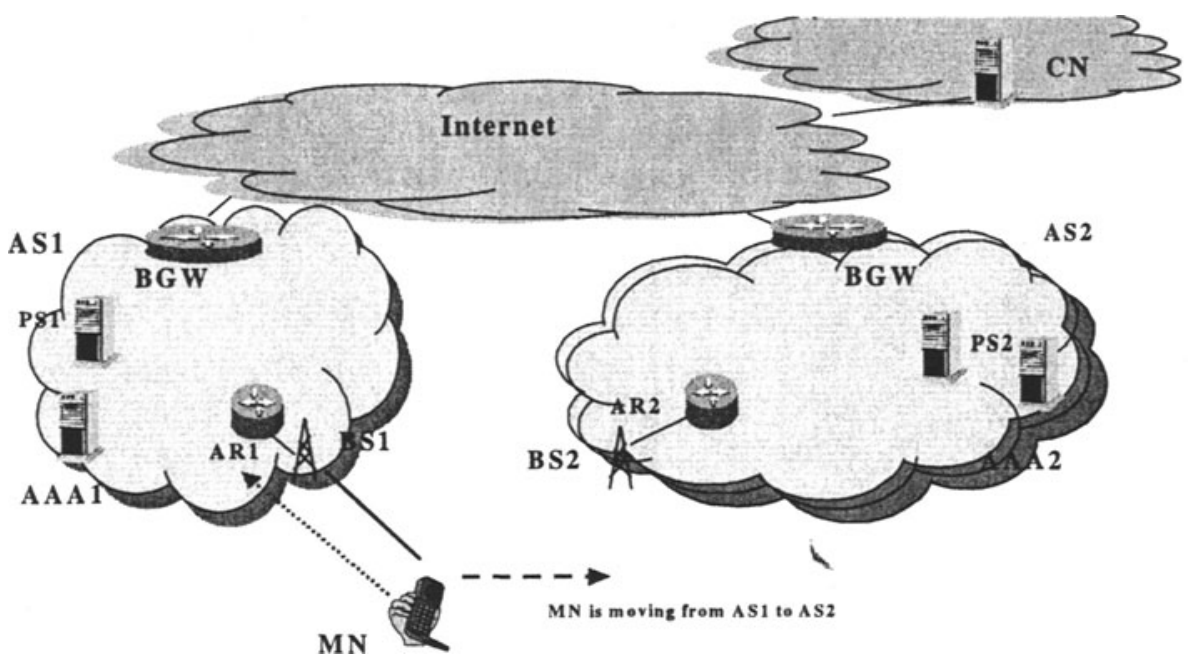

Figure 2 Context Transfer between Access routers

In the following proposed access router selection and context transfer process, policy servers in two different administrative domains may need to exchange information. However, policy servers are typically kept in private address space in each domain for security reasons. One possible solution is to have peering relationships with adjacent administrative domains. Policy servers in different domains can then communicate through border gateway protocol in a secure manner.

\subsection{Selection of access routers prior to handover}

In this selection process, the policy server in the same domain as the candidate access routers computes the selection process. When the MN receives new identifiers from more than one base stations through the broadcast channel, it forwards the information to the AR currently serving it (say AR1). The access router forwards it to the policy server (say PS1). The minimal information which the policy server expects is the either the link layer ID or AS number and link layer ID. If the policy server receives only link layer ID, it first checks the policy database to see whether this is simply an intra domain handover. If it is not an intra domain handover, it queries the neighboring AS defined in the policy database and forwards the link layer ID to the policy server in the neighboring AS.

It would be faster if the AS number is also sent in the broadcast channel by each base station because this eliminates lots of processing. If AS number is sent, the policy server (say PS1) forwards the information to the respective 
policy server (say PS2) along with the MN static capabilities it retrieved from AAA1 server.

After receiving the information, PS2 determines whether its AS can potentially serve the MN. If yes, it further computes the candidate ARs that will be able to serve the MN. PS2 then returns the computed ARs to PS1 (this is totally dependent on the topology of the AS). An algorithm for selecting the ARs is described in the next subsection. If the AS cannot serve the MN, PS2 simply sends a negative acknowledgement to PS1. If MN has forwarded more than one AS identifiers to AR1, the PS1 performs the above steps for each AS. Finally PS1 sends a message to MN informing all the possible (or authorized) ARs that may server the MN.

\subsubsection{An algorithm for AR selection within an AS}

The following is an example of an AR selection algorithm that may be used by a policy server. It is based on a sequence of elimination processes.

Given the set of reachable access routers for the mobile node

1. Eliminate those routers that cannot meet the mobile node's static capabilities

2. Eliminate those routers whose traffic load is above a given threshold

3. Use other operator-defined rules to eliminate more routers

4. Finally, when all rules are executed and if several routers survived, forward all of them to the initiating PS.

The order of the rules may be changed on the policy server. In general, a rule that is able to eliminate more routers should be evaluated before those that eliminate fewer routers. Some time it may be difficult to predict which rule will eliminate more routers. The insight can be gained with experience and a careful analysis of log data on policy servers.

There is no need to reserve any resource at ARs during this process. PS can pre-authorize MN. The initiating PS has to periodically inform the MNs in its network. The second step in the above selection algorithm requires the policy server to have the knowledge of traffic load on access routers. There are two possible ways a policy server may obtain the current load of access routers:

- If the policy server also performs admission controls, it knows the load on those routers naturally. On the other hand, if there is an admission control server, for example a bandwidth broker, the policy server can do a simple query of the server to get the load situation on those routers.

- Some networks may have no centralized admission control. For example, a network may operate on constrained routing where unused network resources are advertised globally within an administrative domain and each router makes admission control 
decision based on the advertised information. In this case, the policy server simply listens the advertisement to know the unused resources on those routers. It then uses that information as an input to the above router selection algorithm.

\section{POLICY BASED CONTEXT TRANSFER}

After going through the router selection process described in the above section and when a handover occurs, an $\mathrm{MN}$ can perform two types of handovers, namely reactive and proactive. In the reactive case, the $\mathrm{MN}$ informs the new access router to pickup its context from the old access router. In the proactive case, the $\mathrm{MN}$ forwards the new access router's identities to the old access router and informs the old access router to push the context to the new access router.

\subsection{Reactive Handover}

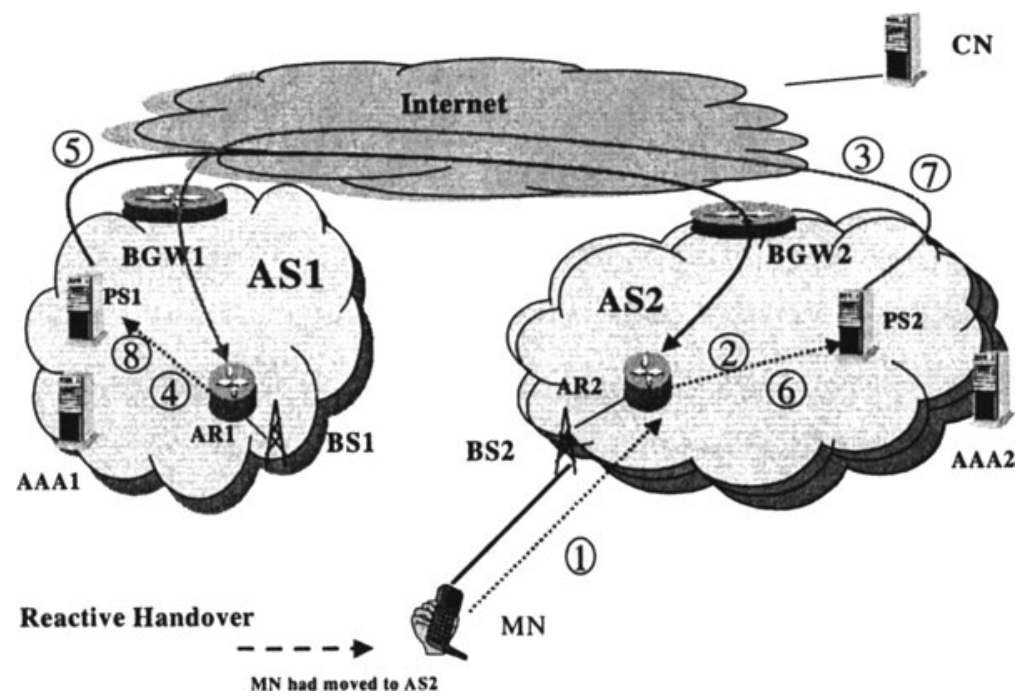

Figure 3 Reactive Handover 


\section{Conditions prior handover:}

- $\mathrm{MN}$ is initially in AS1 and is moving towards AS2. MN picked up more than one base station signals. With the target access router selection process described above, the $\mathrm{MN}$ is aware of the possible access routers who can satisfy its capabilities.

- During the target access router selection process, each possible AS domain has pre-authorized the MN. Hence, context transfer is just a simple relocation of state information.

- Typically, the policy server is totally kept in private address space due to security reasons and is not accessible /visible from outside AS.

In Figure $3 \mathrm{MN}$ that was roaming in the AS1 has moved toAS2. The following message flows occur for the context transfer:

1. MN forwards the AS1:AR1 identity to the AR2.

2. AR2 requests $\mathrm{PS} 2$ to prepare the context transfer request.

3. PS2 forwards the MN context transfer request to the AR1,

4. AR1 sends the context related to the MN to PS1.

5. PS1 adds to the context received from AR1 the static context about MN that is available at PS1. In addition, PS1 may collect other dynamic context from other network elements. For example, MN may have a security context associated with a gateway in AS1. PS1 sends all these static and dynamic contexts to AR2.

6. AR2 extracts the context relevant to AR2 and forwards the rest of the context to PS2.

7. PS2 extracts the static context and forwards the rest of the context to related network elements, e.g., a security gateway that will reconstruct the security context. PS2 sends a context transfer complete message to AR1.

8. AR1 forwards the context transfer complete message to PS1and finally context is removed from AS1.

\subsection{Proactive Handover}

\section{Conditions prior handover:}

1. $\mathrm{MN}$ is initially in the AS1 and is moving towards AS2. MN picks up more than one base station signals. With the target access router selection process described above, the $\mathrm{MN}$ is aware of the possible access routers who can satisfy its capabilities. 
2. During the target access router selection process, each possible AS domain has pre-authorized the MN. Hence, context transfer is just a simple relocation of state information

Figure 4 shows the following message flows:

1. MN that is currently roaming in AS1 decides to move to AS2 because the signal from AS2 is stronger than that from AS1. Under this situation MN forwards the AS2:AR2 identity to the AR1 and requests it to start the context transfer.

2. AR1 forwards the context to PS1 and informs PS1 to forward the context to AR2.

3. PS1 adds to the context received from AR1 the static context about MN that is available at PS1. In addition, PS1 may collect other dynamic context from other network elements. For example, $\mathrm{MN}$ may have a security context associated with a gateway. PS1 sends all these static and dynamic contexts to AR2.

4. AR2 extracts the context relevant to AR2 and forwards the rest of the context to PS2.

5. PS2 extracts the static context and forwards the rest of the context to related network elements, e.g., a security gateway that will reconstruct the security context. PS2 sends a context transfer complete message to AR1.

AR1 forwards the context transfer complete message to PS1 and finally context is removed from AS1.

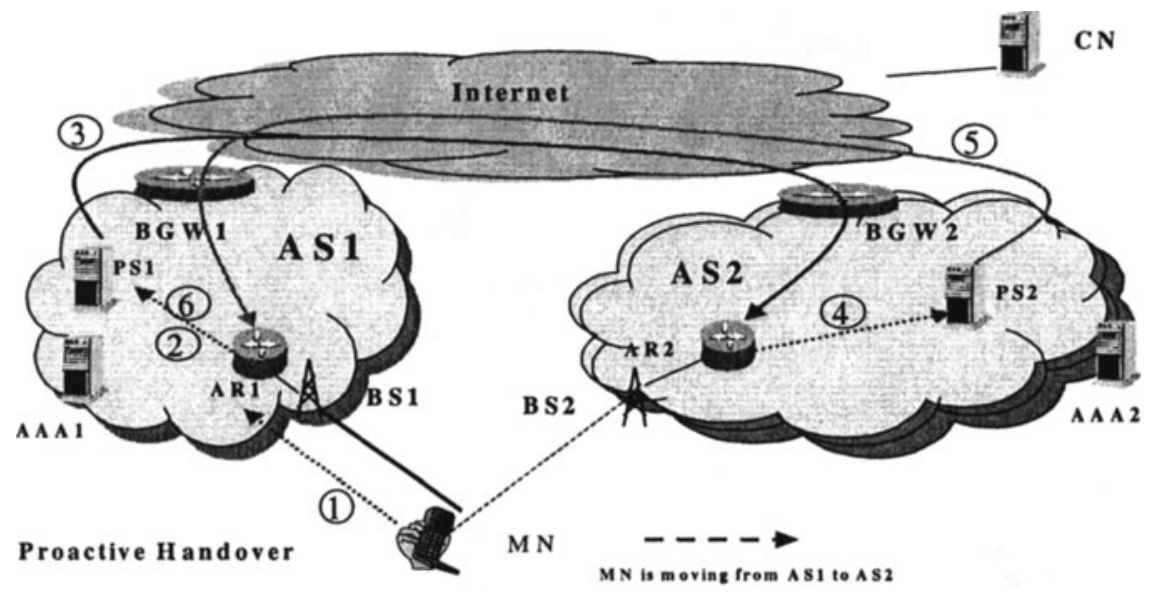

Figure 4 Proactive Handover 


\section{ANALYSIS}

We proposed a policy based target router selection and context transfer approach. It provides the following benefits:

- Balance the functionalities between policy servers and access routers.

- Centralize the critical information for admission control and operation of the network in the policy server. This makes updating and protecting the policy rules easier.

- Routers do not need to expose and discover the capabilities of other routers because this information is readily available at policy servers. This effectively reduces the amount of messages exchanged over the network, saving valuable bandwidth.

- Access routers are freed from target router selection process and most of context transfer process. Hence they can focus on performing their main duty, i.e., route IP packets.

- Access routers need not execute proxy application if their neighbors are running different technology.

- Receiver driven approach for target selection helps in reducing denial of service attacks.

- In most wireless networks, the critical resource is radio spectrum. In the traditional mechanism where handover occurs prior to authorization, radio resources are allocated to the $\mathrm{MN}$ prior to authorization. If the $\mathrm{MN}$ failed the authentication and authorization process, then such radio resources have to be revoked. This could have repercussions such as the blocking of a legitimate user that could be handed over to this network otherwise. By performing an authorization prior to handover, we avoid this problem of blind radio resource allocation, thereby maximizing the capacity of the system.

A possible disadvantage of the proposed solutions is that the policy server may become a single point of failure. Building hardware redundancy into a policy server can solve this problem. In addition, multiple policy servers may be deployed at different locations that will enable both load sharing and fail over. Both solutions are typical ISP practices that have been used, for example, to ensure the availability and reliability of "router reflector'[6].

\section{CONCLUSION}

In this paper we described the problem relating to seamless handover and proposed policy based mechanisms for selecting target access routers and for 
transferring context to the target routers. Our analysis concludes that the policy-based mechanisms are efficient, scalable for network controlled handoff and do not require significant additional functions being built into access routers. In addition, they are secure in the sense that they do not require each administrative domain to expose its interior network topology.

\section{REFERENCES}

1. Dirk Trossen, et al "Issues in candidate access router discovery for seamless IP-level handoffs" IETF Seamoby Working group, http://www.ietf.org/internet-drafts/draft-ietf-seamobycardiscovery-issues-01.txt

2. Dirk Trossen, et al, "Protocol for candidate access router discovery for seamless IP-level handovers", IETF Seamoby Working group individual submission, http://www.ietf.org/internet-drafts/draft-trossen-seamobycardiscovery-00.txt

3. D. Durham, et al "The COPS (Common Open Policy Service) Protocol," IETF RFC 2748, January 2000.

4. M. Fine, et al, “ Differentiated Services Quality of Service Policy Information Base" IETF Diffserv working group. http://www.ietf.org/internet-drafts/draft-ietf-diffserv-pib-05.txt.

5. M. Li, et al, “IPSec Policy Information Base” IETF IPSP working group. http://www.ietf.org/internet-drafts/draft-ietfipsp-ipsecpib-03.txt.

6. T. Bates, R. Chandra, "BGP Router Reflection", RFC 1966, Internet Engineering Task Force, June 1996. 